\title{
Development of a CELestial Infrared Nuller Experiment (CELINE) for broadband nulling and new single-mode fiber testing
}

\author{
Charles Hanot ${ }^{a}$, Pierre Riaud ${ }^{a}$, Dimitri Mawet ${ }^{b}$, Olivier Absil $^{a}$, Jean Surdej $^{a}$ and Serge \\ Habraken ${ }^{c, d}$ \\ ${ }^{a}$ Institut d'Astrophysique et de Géophysique, University of Liège, 17 allée du 6 Août, B-4000, \\ Sart Tilman, Belgium; \\ ${ }^{b}$ Jet Propulsion Laboratory, California Institute of Technology, 4800 Oak Grove Drive, \\ Pasadena, California 91109, USA; \\ ${ }^{c}$ HOLOLAB , University of Liège, 17 allée du 6 Août, B-4000, Sart Tilman, Belgium; \\ ${ }^{d}$ Centre Spatial de Liège, Avenue du Pré-Aily, B-4031, Liège-Angleur, Belgium
}

\begin{abstract}
The small angular distance ( $<100$ mas) and the huge flux ratio $\left(10^{7}\right)$ between an Earth-like exoplanet in the socalled habitable zone and its host star makes it very difficult to direct image such systems. Nulling interferometry consists of a very powerful technique that combines destructively the light from two or more collectors to dim the starlight and to reveal faint companions in its vicinity. We have developed a new nulling experiment based on the fiber nuller principle. ${ }^{1,2}$ This fully symmetric reflective nulling bench aims at testing broadband nulling in both $\mathrm{H}$ and $\mathrm{K}$ bands as well as characterizing photonic fibers for modal filtering. We present in this paper the design, the development as well as preliminary results of the experiment.
\end{abstract}

Keywords: Nulling interferometry, Nulling coronagraphy, single-mode fibers, Achromatic phase shifters, Exoplanets

\section{INTRODUCTION}

Nowadays, in astrophysics, a large number of topics requires to take images of faint structures located at very small angular distances from much brighter sources. Among them, extra-solar planet imaging and characterization is a subject that draw a lot of attention since the discovery of the first exoplanet around a solar type star 15 years ago. ${ }^{3}$ Since then, the scientific community has been obsessed by the idea of imaging an earth-twin hosting life around another star. Unfortunately for us, such a "picture" is very difficult to take as the flux ratio between the exoplanet and its parent star is about $10^{-7}$ in the thermal infrared and $10^{-10}$ in the visible. Moreover, the angular separation between them is also a critical aspect as it is smaller than $0.1^{\prime \prime}$, even for the nearby stars. One of the solution that has been found to overcome these two issues is nulling interferometry. ${ }^{4}$ The principle is to recombine destructively the light coming from different apertures in order to cancel the on-axis starlight and reveal the presence of faint companions in its vicinity. A lot of research and development has already been done on the topic and deep broadband starlight rejection (also called null depth) down to $10^{-5}$ have been obtained in the lab at thermal infrared wavelengths $([8-12 \mu \mathrm{m}]) .{ }^{5}$ This results prove the feasibility of Earth-like exoplanet detections with spaceborne infrared nulling interferometers. Unfortunately, ground based interferometers are strongly perturbed by atmosphere turbulences and many develoments are still required to perform ground based interferometric observations at very high contrast. Today, the developments of ground based nulling are led by two instruments: the Keck Interferometer Nuller ${ }^{6,7}$ and the Palomar Fiber Nuller ${ }^{1,2}$ with starlight suppressions of a couple of $10^{-4}$. $^{8}$

The nulling interferometer experiment that we develop at University of Liège is called CELINE for CELestial Infrared Nulling Experiment. Its goal is do develop key technologies for actual or future ground based nulling

Further author information: (Send correspondence to Charles Hanot)

Charles Hanot: E-mail: hanot@astro.ulg.ac.be, Telephone: +32 (0)4 3669768

Optical and Infrared Interferometry II, edited by William C. Danchi, Françoise Delplancke, Jayadev K. Rajagopal, Proc. of SPIE Vol. 7734, 77342L · (c) 2010 SPIE · CCC code: 0277-786X/10/\$18 · doi: 10.1117/12.856843 


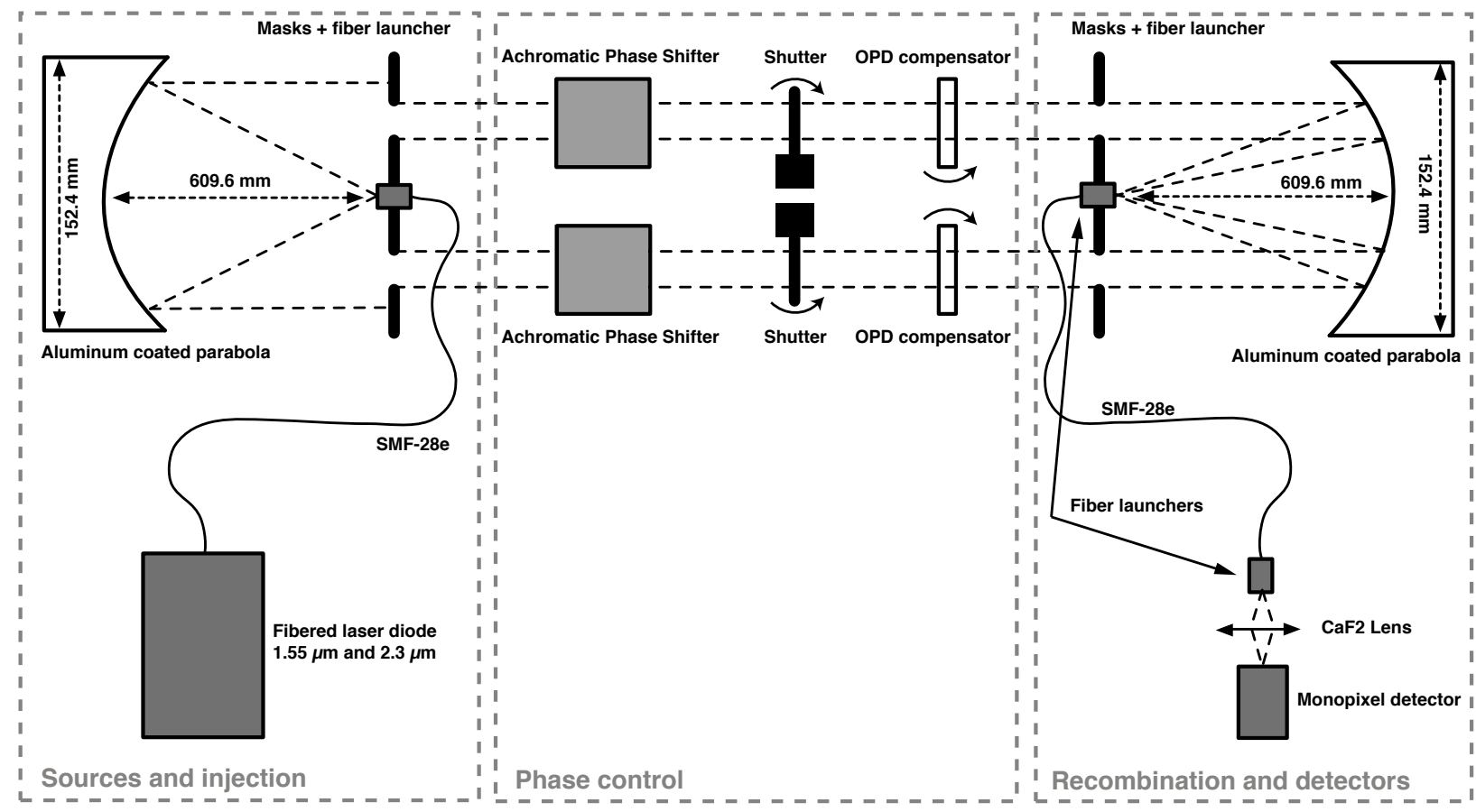

Figure 1. Layout of CELINE nulling testbench. The laser source is injected into a single mode fiber and directly feeds the nuller. After being collimated by a 6 inch parabola, the main beam is divided into two circular aperture of $10 \mathrm{~mm}$ separated by $60 \mathrm{~mm}$. The two interferometric arms are then phase shifted by a halfwave. This step is done by combining tilted Infrasil 301 parallel plates together with achromatic phase shifters (Waveplates or Fresnel rhombs). The beams are then sequentially blocked by two shutters in order to measure the 4 different interferometric signals (individual beam intensities, interferometric signal and background intensity). After passing through another pupil mask, identical to the first one, the beams are focused into a single mode fiber which acts both as a beam combiner and as a modal filter. The output of this fiber is finally focused onto a monopixel InGaAs infrared detector.

facilities in the near and mid infrared (H, K and L bands) such as the Palomar Fiber Nuller or ALADDIN. ${ }^{9}$ In this paper, we present the instrumental design as well as preliminary results obtained with the testbench in the lab.

\section{INSTRUMENTAL DESCRIPTION}

The main driver of the nulling interferometer design we present in this paper is its simplicity and versatility. Indeed, the simpler the design, the less degree of freedom one has to constrain during the alignment of the system and the less sources of errors are present in the instrument. For this reason, the CELINE instrumental layout is based on the off-axis single mode fiber beam recombination. ${ }^{1}$ Such a beam recombination technique has been used in different interferometers such as the Palomar Fiber Nuller ${ }^{1,2}$ and MAII ${ }^{10}$ and has proven its capabilities. Indeed, broadband ( $20 \%$ bandwidth) null depth of $\sim 10^{-4}$ have been obtained in the H-band. ${ }^{11}$ The principle is to recombine the different interferometric arms by directly focusing them into a common single mode fiber. This technique strongly reduces the optical complexity of interferometers as the single mode fiber is used both as a beam combiner and a single-mode filter.

Our instrumental layout is entirely designed to optimize this off-axis recombination technique and is illustrated by Fig. 1. The light source feeds the interferometer through a single mode fiber. An on-axis parabola first collimates the beam which is then blocked by a mask which defines two circular apertures. These two apertures have a diameter of $10 \mathrm{~mm}$ and are separated by $60 \mathrm{~mm}$. The pupil mask is not adjustable (size of apertures, baseline, etc..) and is attached to the input fiber launcher in order to reduce the number of degrees of freedom. Once these two interferometeric beams are defined, OPD compensators that consist in tilted parallel plates take care of co-phasing the two arms. Two shutters then sequentially blocks the beams in order to have access to 


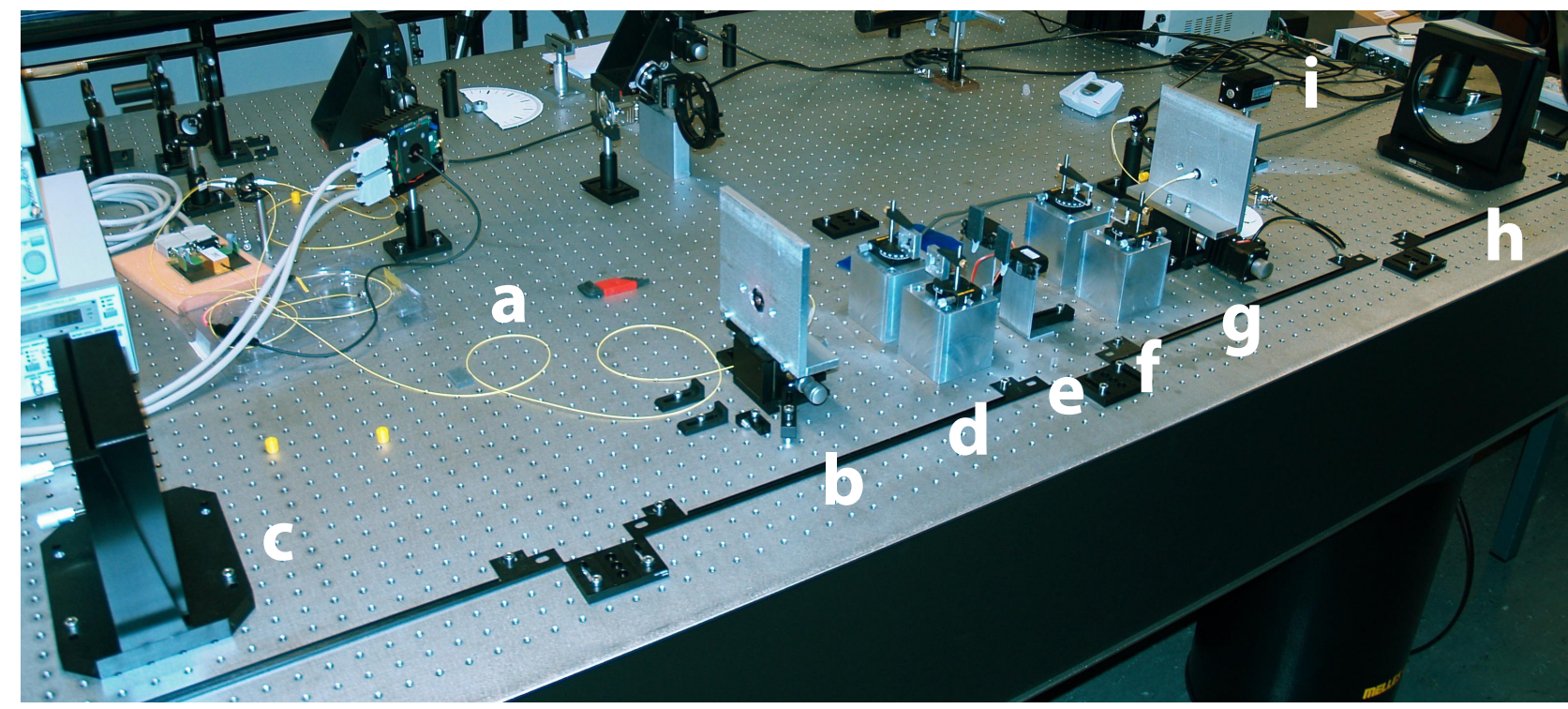

Figure 2. Picture of the CELINE testbench. Following the light path, you can see: (a) the input single mode fiber (b) the pupil mask and fiber launcher (c) the 6 inch parabola (d) the achromatic waveplates (e) the two shutters (f) the tilted Infrasil 301 parallel plates (g) the second pupil mask and the output fiber fiber launcher (h) the second 6 Inch parabola and (i) the mono-pixel detector.

the individual beam intensities, the background level and the interferometric signal. Achromatic phase shifters designed for specific wavebands are then inserted to induce a relative $\pi$ phase shift between the two channels. Finally, after a new pupil mask that gets rid of some diffracted light (Fresnel propagation), the two beams are focused by a parabola into the output single mode fiber. Finally after being filtered by the fiber, the output signal is focused onto a mono-pixel detector.

This testbench, although very simple has a lot of qualities:

- The number of optical elements is minimized with only two reflective mirrors. Therefore, it increases the optical wavefront quality and limit the throughput losses.

- The optical layout has a high degree of symmetry and the beams share the same optics and are focused into the same single mode fiber. Therefore, the non common path errors are minimized (no polarization rotation, no chromatism effects from coating differences, etc...).

Now that the overall description of the testbench has been done let us explain in more details the three main blocks that compose the instrument (See Fig. 1).

\subsection{Sources and injection}

Four different laser sources are available with wavelengths ranging from $450 \mathrm{~nm}$ to $2500 \mathrm{~nm}$ : (i) a $650 \mathrm{~nm}$ diode that is used for alignment (ii) a $1550 \mathrm{~nm}$ diode (iii) a $2300 \mathrm{~nm}$ diode and (iv) a $2 \mathrm{~W}$ supercontinuum white light source with operational wavelengths between $\sim 450 \mathrm{~nm}$ to $\sim 2500 \mathrm{~nm}^{*}$. While the $650 \mathrm{~nm}$ laser diode is only used for alignment purposes, the two other monochromatic diodes (1550 nm and $2300 \mathrm{~nm}$ ) are used for testing monochromatic rejections in $\mathrm{H}$ and $\mathrm{K}$ bands. Finally the supercontinuum white ligh source will be coupled with $\mathrm{H}$ and $\mathrm{K}$ filters to perform broadband ( $\sim 20 \%$ bandwidth) nulling. All these sources are fiber-coupled into a SMF 28e single mode fiber. This fiber has a cutoff wavelength $<1260 \mathrm{~nm}$ and works up to the $\mathrm{K}$ band. Unlike the classical SMF-28 and most single-mode fibers designed for telecom applications, the SMF-28e fiber is designed to minimize $\mathrm{OH}$ absorption lines. For longer wavelengths however, the filtering capabilities decrease and the

\footnotetext{
*This source is currently not available on the bench but is planned to be operational by the end of 2010
} 


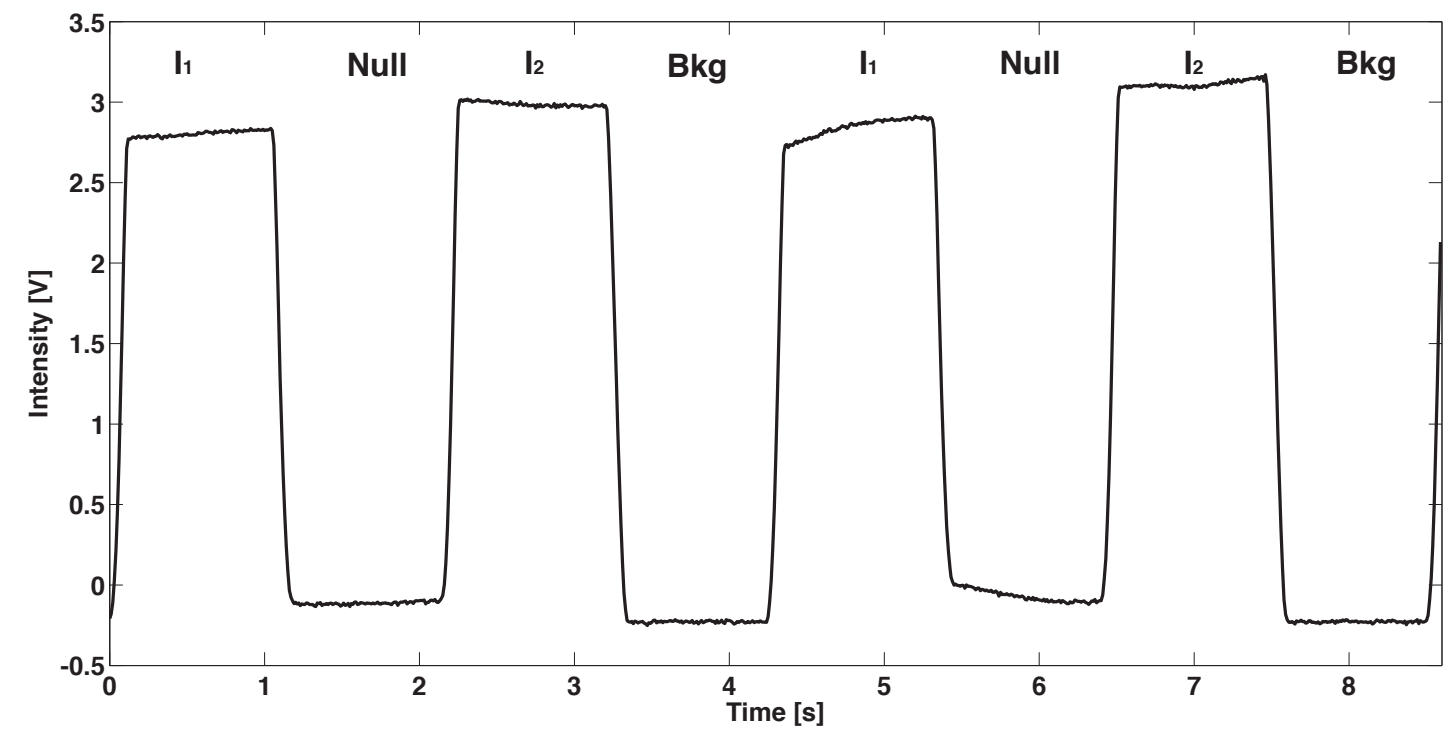

Figure 3. Chopped signal measured by the detector during two chop cycles of $4.25 \mathrm{~s}$. The four chopping states are clearly visible with successive measurements of beam 1 intensity, of the interferometric (or nulling) signal, of beam 2 intensity and of the background intensity.

fiber starts to absorb. However, for our current applications in both $\mathrm{H}$ and $\mathrm{K}$ bands, the SMF-28e is sufficiently transparent and allows us to quickly switch between the different light sources without doing any additional adjustment. Later on, to optimize the performances and the throughput in $\mathrm{K}$ band, we plan on upgrading the testbench with fluoro-zyrconate monomode fibers specially designed for our instrument.

The fiber end is mounted on a three axis flexture mount that provides a micrometer injection accuracy. The light injected into the bench is then directly collimated by a 6 inches parabola having a $\mathrm{f} / 4$ focal ratio. This focal ratio corresponds to the numerical aperture of the SMF-28e fiber. The reason for choosing such a large parabola is for versatility purposes. Indeed, one of the testbench main goals is to the test Fresnel rhombs achromatic phase shifters $^{12,13}$ (see Sect. 2.2) which requires a minimum spacing of $60 \mathrm{~mm}$ between the beams. After the collimation, it passes through a pupil mask that produces two circular apertures of $10 \mathrm{~mm}$. The pupil mask and the fiber launcher are on a common mount to reduce the number of optical elements to be alignmed.

\subsection{Phase control and signal chopping}

The central part of the interferometer is the cornerstone of the instrument as it contains the optical components that control the differential phase between the interferometer arms and the device used to recover the different interferometric signals.

Indeed, the drawback of interferometers using off-axis single mode beam recombination is that there is no direct access to the individual beam intensities and to the background level. A piece of hardware that we describe in this section must therefore be used to have access to this information. This device consist in two masks mounted on servo-motors blocking sequentially each interferometric beam. The frequency at which these motors operate can be tuned between a fraction of Hertz and 10Hz. Fig. 3 shows an exemple of chopped signals recorded by the nuller. The four different signals (i.e. the individual intensities, the background and the interferometric signal) are clearly visible over two chop cycles of $\sim 4.25$ second each.

One of the most critical part of a nulling interferometer is certainly the phase shifters. Indeed, from the accuracy to which the relative phase shift between the two beams is introduced depends the null depth that you can reach. 
The best achievabe polychromatic null depth $N$ depends quadratically on the phase error:

$$
N=\frac{\int_{\lambda_{\min }}^{\lambda_{\max }} \Delta \phi(\lambda)^{2} \eta(\lambda) \mathrm{d} \lambda}{4 \int_{\lambda_{\min }}^{\lambda_{\max }} \eta(\lambda) \mathrm{d} \lambda}
$$

where $\lambda$ is the wavelength, $\lambda_{\min }$ and $\lambda_{\max }$ are respectively the minimum and maximum wavelength of the instrument waveband, $\Delta \phi(\lambda)$ is the phase shift error and $\eta(\lambda)$ is the spectral instrumental response

On our tesbench, the first step for phase control is to match the two beams optical paths by inserting in each beam path an Infrasil 301 parallel plate. These two plates have been polished together to minimize their thickness difference $(<150 \mathrm{~nm})$ and are polished with a flatness better than $\lambda / 10$ peak to valley. By tilting one of the two plates, the optical path through Infrasil changes. It is therefore possible to accurately match the OPD. Because the CELINE design is very symmetric, it is not necessary to use more complex delay lines as a proper alignment of the nulling bench leaves very small OPD residuals already. Indeed, first measurements in the lab show that the differential optical path between the two beams is $<10 \mu \mathrm{m}$. The second step consists in introducing the relative $\pi$ phase shift between the two beams. This phase shift, together with the OPD compensation must be as close to $\pi$ as possible over the whole waveband (see Eq. 1). For that, two achromatic phase shifter (APS) designs are planned to be used : (i) achromatic waveplates and (ii) Fresnel rhombs.

Waveplates are optical elements which introduce a relative phase shift between the polarization components $s$ and $p$ of the incident light. For example, parallel plates made of birefringent materials have such properties. The achromatic halfwave plates that we use for CELINE are made of two waveplates and have been optimized to provide an achromatic $\pi$ phase shift over the $\mathrm{H}$ band. By combining Quartz and MgF2 waveplates with respective thicknesses of $1.5268 \mathrm{~mm}$ and $1.199 \mathrm{~mm}$ it is possible to reach Null Depthsx down to $\sim 4 \times 10^{-5}$ over the full H-band.

Fresnel rhombs are optical grisms that make use of total internal reflection to introduce a phase shift between the vectorial $s$ and $p$ polarization components. Classical Fresnel rhombs are limited by the intrinsic index dispersion of the rhomb bulk material. ${ }^{14}$ Engraving a subwavelength grating (often called zero order grating, or ZOG) on the TIR interface or simply depositing a single thin layer of a well chosen material leads to a significant improvement. ${ }^{15,16}$. The Fresnel rhombs we use for our experiment are made of ZnSe and have been optimized for the thermal infrared, and especially for the wavelength range $[6-11] \mu \mathrm{m}$. Even then, its phase shifting capability in both $\mathrm{H}$ and $\mathrm{K}$ bands is sufficient to reach Null Depths down to $\sim 10^{-4}$ at these wavelengths.

\subsection{Recombination and detector}

After having been phase shifted with respect to each other, the two interferometric beams pass through a new mask which is strictly identical to the mask defining the pupils. This mask gets rid of some diffracted light. Then, the two beams are focused into the single mode fiber by a 6 inch parabola. The couple parabola/fiber is the same one for the front end of the bench as for the back end. The horizontal movement of the 3 axis flexure mount on which the fiber mount is placed is controled by a stepper motor. The stepper motor control the alignment of the fiber used for the beam recombination with an accuracy of $39 \mathrm{~nm}$. This stepper motor is currently operated manually but will soon work in close loop to optimize the instrumental null depth in real time. Finally, the output of the single mode fiber is focused onto a Hamamatsu In GaAs mono-pixel detector that is sensitive up to $2.56 \mu \mathrm{m}$.

\section{RESULTS}

\subsection{Data analysis}

The data analysis method we use in this paper is explained in details by Hanot et al., 2010. ${ }^{8}$ The principle is to use the nulling depth histogram to retrieve the astrophysical information despite the instrumental fluctuating noise. In a lab with a point-like source, a nuller should theoretically give an astrophysical null $N_{a}=0$. Any departure from 0 shows the instrumental limitation of the instrument that are not corrected by the statistical reduction method we use.

The shutters (see Fig. 3) allow us to measure successively the individual beam intensities $I_{1}(t)$ and $I_{2}(t), I_{B}(t)$ 
the background intensity and $I_{-}(t)$ the combined intensity of the two beams "at null" with frequencies ranging from fraction of Hertz up to 10 Hertz. The observed null depth is normally defined as follows

$$
N_{\mathrm{Ob}}(t)=\frac{I_{-}(t)-I_{B}(t)}{I_{+}(t)-I_{B}(t)}
$$

where $I_{+}(t)$ is the interferometric signal measured on the central constructive fringe ("at max"). However, in the case of single-mode fiber beam combiner based interferometers, the simultaneous access to the different intensities of Eq. 2 is not possible and the observed null depth for each chop cycle is

$$
N_{\mathrm{Ob}}(t)=\frac{I_{-}(t)-\left\langle I_{B}(t)\right\rangle}{\left\langle I_{+}(t)\right\rangle-\left\langle I_{B}(t)\right\rangle}
$$

where

$$
\begin{aligned}
\left\langle I_{B}(t)\right\rangle & =\frac{1}{\Delta t} \int_{t_{0}+\Delta t}^{t_{0}+2 \Delta t} I_{B}(t) \mathrm{dt} \\
\left\langle I_{+}(t)\right\rangle & =2\left[\left\langle I_{1}(t)\right\rangle+\left\langle I_{2}(t)\right\rangle\right] \\
& =\frac{2}{\Delta t}\left[\int_{t 0-2 \Delta t}^{t 0-\Delta t} I_{1}(t) \mathrm{dt}+\int_{\mathrm{t} 0-\Delta \mathrm{t}}^{\mathrm{t} 0} \mathrm{I}_{2}(\mathrm{t}) \mathrm{dt}\right]
\end{aligned}
$$

$t_{0}$ being defined as the first epoch of a chop cycle starting with the measurement of $I_{-}(t)$ and $\Delta t$ the length of a chopping state corresponding to a quarter of the chopping period. From these definitions, at every time $t$ within a chop cycle, we have $t=t_{0}+\delta t$.

Unfortunately, in presence of fluctuating noise sources such as phase errors $\left(\Delta \phi_{d}(t)\right)$ and intensity mismatch $(\delta I(t))$, the observed null depth varies quickly as a function of time. The dependence of the null depth on these error sources is

$$
N(t)=\frac{\Delta \phi_{d}(t)^{2}}{4}+\frac{\delta I(t)^{2}}{16}+N_{a}
$$

where $N_{a}$ is the astrophysical null depth. Moreover, for interferometers that do not measure simultaneously the different channels, one must add to Eq. 7 other noise contributors ${ }^{8}$

$$
N(t)=I_{N}(t, \Delta t)\left[\frac{\Delta \phi_{d}(t)^{2}}{4}+\frac{\delta I(t)^{2}}{16}+N_{a}\right]+N_{B}(t)
$$

where $I_{N}(t, \Delta t)=2\left(I_{1}(t)+I_{2}(t)\right) /\left\langle I_{+}(t)\right\rangle$ is the normalized intensity uncertainty and $N_{B}(t)=\left(I_{B}(t)-\right.$ $\left.\left\langle I_{B}(t)\right\rangle\right) /\left\langle I_{+}(t)\right\rangle$ is the equivalent background null depth. These fluctuating noise sources make much harder the accurate determination of the astrophysical null depth. The aproach proposed by Hanot et al. $2010^{8}$ consist in fitting the histogram corresponding to Eq. 8 by a model using the intensities and phase distributions to recover the astrophysical information.

Indeed, with CELINE, $\delta I(t), I_{N}(t, \Delta t)$ and $N_{B}(t)$ distributions are monitored. Therefore, the nulling histogram of Eq. 8 only depends on the phase error distribution $\Delta \phi_{d}(t)$ and on the astrophysical null depth $N_{a}$. If we consider that $\Delta \phi_{d}(t)$ follows a normal distribution, it is then possible to fit a nulling histogram model having only 3 free parameters : $\mu_{\phi}$ the mean phase error, $\sigma_{\phi}$ its standard deviation and $N_{a}$. This technique allows to reach a much better accuracy and sensitivity on the measurement of the astrophysical null depth as we are not limited by the mean errors like in most data reduction methods but by the uncertainties on their distribution. Moreover, for on sky observations, it has the advantage of not requiring calibration sequences. In the lab, using a point like source generated by a laser that is injected into a single-mode fiber, the measured astrophysical null depth is supposed to be 0 . Therefore, any departure of $N_{a}$ from zero traduce an instrumental bias that is not compensated by the reduction method 

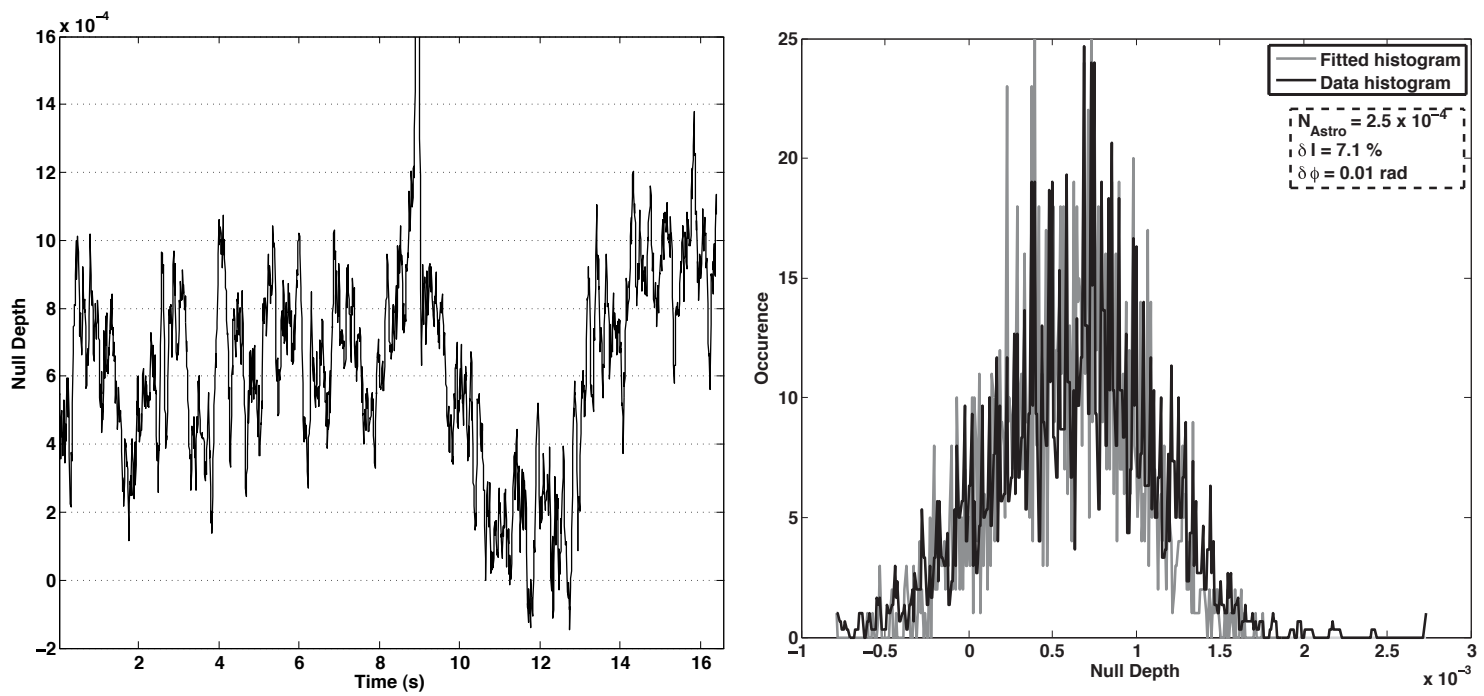

Figure 4. Left: Nulling sequence of 16 s obtained with the $1550 \mathrm{~nm}$ monochromatic laser source. During the measurement, the null depth averaged over periods of $1 \mathrm{~s}$ is constantly below $10^{-3}$. Between the 10th and 13th seconds, the null depth even dropped down to $2 \times 10^{-4}$. Right: Null depth distribution corresponding to the left panel sequence. The black curve represents the measurement distribution while the grey curve is the best fit of the nulling distribution. It corresponds to a null depth of $2.5 \times 10^{-4}$.

\subsection{Nulling results with monochromatic light}

The CELINE testbench is in its building and debugging phase. The results that we present here have been obtained with the very first light of the instrument and do not represent the final limitations of our experiment. They have been obtained with the $1550 \mathrm{~nm}$ laser diode during the alignment phase of the testbench. Figure 4 (left) show a nulling sequence of $16 \mathrm{~s}$. During these $16 \mathrm{~s}$, the measured mean null depth is constantly below $10^{-3}$. The Null Depth even dropped down to $1.8 \times 10^{-4}$ during $3 \mathrm{~s}$. Over longer timescales, current measurements show a slow drift of the null depth. This drift comes from a relaxation of the three axis flexure mount holding the recombination fiber along the horizontal axis. It is however possible to control this drift by adjusting the stepper motor.

Figure 4 (right) represent the null depth distribution during this nulling sequence. The black curve corresponds to the measured distribution while the grey one is the best fitted distribution. The fitted null depth corresponding to this distribution is $2.5 \times 10^{-4}$. The mean intensity mismatch and phase errors during this sequence are respectively $\delta I=7.1 \%$ and $\mu_{p h i}=0.01 \mathrm{rad}$. The limitation that we observe during our measurement at a couple of $10^{-4}$ is fully consistent with the measured intensity mismatch. Indeed, the effect of intensity mismatch on the null depth is given by $\delta I^{2} / 16 \simeq 3 \times 10^{-4}$. One way to increase the performances and the stability of CELINE will be to control the injection of the beams into the single-mode fiber in real time using the stepper motor (see Sect. 2.3).

\section{PERSPECTIVES}

Even though we are still at the early stages of the development of our instrument, the results obtained are already promising with monochromatic null depth down to $2 \times 10^{-4}$ at $1550 \mathrm{~nm}$ with the very first light of the instrument. The objectives we have with the testbench are numerous and will start with an upgrade of the bench with a supercontinuum white light source. This very bright and broadband laser source will allow us to perform broadband measurements in both $\mathrm{H}$ and $\mathrm{K}$ bands at very high contrasts. Our goal is to reach broadband null depths between $\sim 10^{-4}$ and $10^{-5}$ in both of these bands. For that, we will use achromatic waveplates for the $H$ band and Fresnel rhombs for $\mathrm{H}$ and $\mathrm{K}$ bands. For $\mathrm{K}$ band measurements, in order to optimize the throughput and the wavefront filtering capabilities, we will upgrade the bench with fluoro-zyrconate single-mode fibers, specially designed for the use of CELINE in $\mathrm{K}$ band. We are also planning to test the capabilities of photonic fibers for 
off-axis beam recombination. Indeed, such fibers have never been tested as modal filters and beam combiners for nulling interferometry. Indeed, the particularity of these fibers is that they do not have a Gaussian mode like most fibers but a Bessel J1 mode. Their interest, however, comes from their broadband qualities as their typical bandwidth is $\mathrm{R} \simeq 2$.

Finally, the ultimate goal of our testbench will be to use CELINE as a technology demonstrator for future ground based nulling facilities in L band such as ALADDIN. This will require of course a major upgrade of the bench with a new source, new APS, a new detector and of course new single-mode fibers (Chalcogenids).

\section{ACKNOWLEDGMENTS}

The research was supported by the Fond National de la Recherche scientifique de Belgique (FNRS), by the Fonds pour la formation à la Recherche dans l'Industrie et dans l'Agriculture de Belgique (FRIA) and by the Communauté Francaise de Belgique - Action de recherche concertée - Académie Wallonie - Europe. The authors acknowledge Jacques Baudrand (LESIA) and ESA for generously lending us respectively achromatic halfwave plates and Fresnel rhombs.

\section{REFERENCES}

1. Haguenauer, P. and Serabyn, E., "Deep nulling of laser light with a single-mode-fiber beam combiner," Appl. Opt. 45, 2749-2754 (2006).

2. Martin, S., Serabyn, E., Liewer, K., Loya, F., Mennesson, B., Hanot, C., and Mawet, D., "The development and applications of a ground-based fiber nulling coronagraph," in [Society of Photo-Optical Instrumentation Engineers (SPIE) Conference Series], Society of Photo-Optical Instrumentation Engineers (SPIE) Conference Series $\mathbf{7 0 1 3}$ (July 2008).

3. Mayor, M. and Queloz, D., "A Jupiter-Mass Companion to a Solar-Type Star," Nature 378, 355-+ (Nov. 1995).

4. Bracewell, R. N., "Detecting nonsolar planets by spinning infrared interferometer," Nature 274, 780-781 (Aug. 1978).

5. Peters, R. D., Lay, O. P., and Lawson, P. R., "Mid-Infrared Adaptive Nulling for the Detection of Earthlike Exoplanets," PASP 122, 85-92 (Jan. 2010).

6. Serabyn, E., Booth, A. J., Colavita, M. M., Creech-Eakman, M. J., Crawford, S. L., Garcia, J., Johnson, R. L., Hovland, E., Koresko, C., Ligon, E. R., Martin, S. R., Mennesson, B. P., Moore, J. D., Palmer, D. L., Paine, C. G., Shao, M., Swain, M. R., Smythe, R. F., and Vasisht, G., "The Keck interferometer nuller: system architecture and laboratory performance," in [New Frontiers in Stellar Interferometry], Traub, W., ed., Proc. SPIE 5491, 806-815 (Oct. 2004).

7. Colavita, M. M., Serabyn, E., Millan-Gabet, R., Koresko, C. D., Akeson, R. L., Booth, A. J., Mennesson, B. P., Ragland, S. D., Appleby, E. C., Berkey, B. C., Cooper, A., Crawford, S. L., Creech-Eakman, M. J., Dahl, W., Felizardo, C., Garcia-Gathright, J. I., Gathright, J. T., Herstein, J. S., Hovland, E. E., Hrynevych, M. A., Ligon, E. R., Medeiros, D. W., Moore, J. D., Morrison, D., Paine, C. G., Palmer, D. L., Panteleeva, T., Smith, B., Swain, M. R., Smythe, R. F., Summers, K. R., Tsubota, K., Tyau, C., Vasisht, G., Wetherell, E., Wizinowich, P. L., and Woillez, J. M., "Keck Interferometer Nuller Data Reduction and On-Sky Performance," PASP 121, 1120-1138 (Sept. 2009).

8. Hanot, C., Mennesson, B., Serabyn, E., Martin, S. R., Liewer, K., Loya, F., Mawet, D., Riaud, P., and Absil, O., "Improving interferometric null depth measurements with statistics: theory and first results with the palomar fiber nuller," In prep. (2010).

9. Absil, O., Coudé du Foresto, V., Barillot, M., and Swain, M. R., "Nulling interferometry: performance comparison between Antarctica and other ground-based sites," A $\mathscr{G} A$ 475, 1185-1194 (Dec. 2007).

10. Buisset, C., Rejeaunier, X., Rabbia, Y., and Barillot, M., "Stable deep nulling in polychromatic unpolarized light with multiaxial beam combination," Appl. Opt. 46(32), 7817-7822 (2007).

11. Mennesson, B., "A Single-Mode Nulling Rotating Coronagraph for High Contrast Ground Based Imaging," in [In the Spirit of Bernard Lyot: The Direct Detection of Planets and Circumstellar Disks in the 21st Century], (June 2007). 
12. Mawet, D., Hanot, C., Lenearts, C., Riaud, P., Vandormael, D., Loicq, J., Fleury, K., Plesseria, J. Y., Defrère, D., Surdej, J., and Habraken, S., "Fresnel Rhombs as Achromatic Phase Shifters for Infrared Nulling Interferometry," Submitted to Optics Express (2007).

13. Hanot, C., Mawet, D., Loicq, J., Vandormael, D., Plesseria, J. Y., Surdej, J., and Habraken, S., "Fresnel rhombs as achromatic phase shifters for infrared nulling interferometry: first experimental results," in [Society of Photo-Optical Instrumentation Engineers (SPIE) Conference Series], Presented at the Society of Photo-Optical Instrumentation Engineers (SPIE) Conference 6693, 51-+ (Sept. 2007).

14. Anderson, R., "Quarterwaveplate and Fresnel rhomb compared in the 10-Mum CO2 laser emission region," Appl. Opt. 27, 2746-2747 (1988).

15. Mawet, D., Lenaerts, C., Riaud, P., Surdej, J., Habraken, S., and Vandormael, D., "Use of subwavelength gratings in TIR incidence as achromatic phase shifters," œ 13, 8686-8692 (2005).

16. Mawet, D., Lenaerts, C., Riaud, P., Vandormael, D., Loicq, J., Verstraeten, D., Fleury, K., S., H., and Surdej, J., "Infrared achromatic phase shifters using modulated total internal reflection," in [Advances in Stellar Interferometry. Edited by Monnier, John D.; Schöller, Markus; Danchi, William C.. Proceedings of the SPIE, Volume 6268, pp. 62682J (2006).], SPIE, ed., 6268 (2006). 